\title{
Research on Facial Expression Recognition based on Motion Unit Combi- nation Feature Matrix and Supporting Vector Machine
}

\author{
Shi Shui-e $\mathrm{e}^{1, *}$ and Guo Rong-yan ${ }^{2}$ \\ ${ }^{I}$ College of Physics and Electronic Engineering, Henan Normal University, Xinxiang 453007, China \\ ${ }^{2}$ School of Physics and Mechanical \& Electrical Engineering, Zhoukou Normal University, Zhoukou, Henan, 466001, \\ China
}

\begin{abstract}
Based on Haar and Adaboost methods, this paper uses genetic algorithm and cloud computing, collaborative simulation to improve facial expression recognition algorithm. It uses genetic algorithm to encode the movement element local feature combination, which improves marked effect of facial organslocal feature region. It uses cloud computing collaborative simulation topology to establish facial local feature generalized matrix, which enhanced the calculation speed of the support vector machine expression classifier. In order to verify the efficiency and accuracy of the algorithm, this paper tests the facial expressions of the same individuals and different individuals using expression library. Throguh testing it is found that the improved method has higher facial expression recognition rate, faster computing speed and better performance. Throguh the analysis of results, the improved algorithm has higher facial expression recognition rate and it is higher in the same individual and different individuals, and the recognition rate of different individuals is the same as the average recognition rate, which verifies the reliability of the algorithm and provides a new method for the design of facial expression recognition algorithm.
\end{abstract}

Keywords: Haar, Adaboost, Motion unit, Characteristic matrix, Cloud computing, Supporting vector machine.

\section{INTRODUCTION}

Computer is difficult to recognize people compared to human self, the main reasons is that in a recognition process, the computer needs to establish characteristics expression and emotion classification of human facial expression model at the same time, but also they need be connected $[1,2]$. The face is a flexible body, and it is very difficult to associate facial movement with facial expression using a fixed pattern, in addition, the facial expression is diversity. Facial expressions produce subtle changes due to inner emotional, so it is the difficulty of facial expression recognition.

Facial expression recognition algorithm has gone through three stages [3]: the first stage is the face recognition or texture recognition is applied to facial expression recognition, the most commonly used features are Haar feature, LBP feature and Gabor feature, and the most commonly used classifiers are mainly SVM, Adaboost and neural network [4]. For example, Kobayashi used the neural network method for facial expression recognition; Yin Xingyun et al. use the hidden Markov model for facial expression recognition, and good results have been obtained; In the second stage, most of the work is around the unique characteristics of facial expression, such as Donate and Tian proposed motion unit recognition. Combined context information of facial features in the template matching Ding achieves a precise positioning of facial motion unit. The third stage is from two aspects, including information collection and classification $[5,6]$. By simulating the macaque from retina to visual neurons, Gu put forward the local feature extracting based on jurisdiction coding. Through the simulation of biological visual response mechanism, Wright proposed a classification method based on sparse coding, and proved that SRC can solve the occlusion and noise problem in the face recognition.

There are two prominent problems in the system design process of facial expression recognition: one of them is the recognition rate, the other is the calculation speed [7]. Based on this, this paper designs the overall framework of the facial expression recognition, as shown in Fig. (1).

Fig. (1) shows the basic framework of facial expression recognition system designed in this paper. The cloud collaborative computing algorithm is based on multi-core and parallel computing, and it can effectively improve the computational efficiency of the classifier. The genetic algorithm coding can mark on facial motion unit feature, which improves the samples training speed, lowers the accuracy of recognition and reduces error rate [8]. So, genetic algorithm and cloud collaborative computing are the main technologies of system optimization.

\section{FEATURE EXTRACTION METHOD BASED ON FACIAL MOTION UNIT}

Whitehill uses many advanced algorithms in the smile recognition method and different algorithms are compared, 


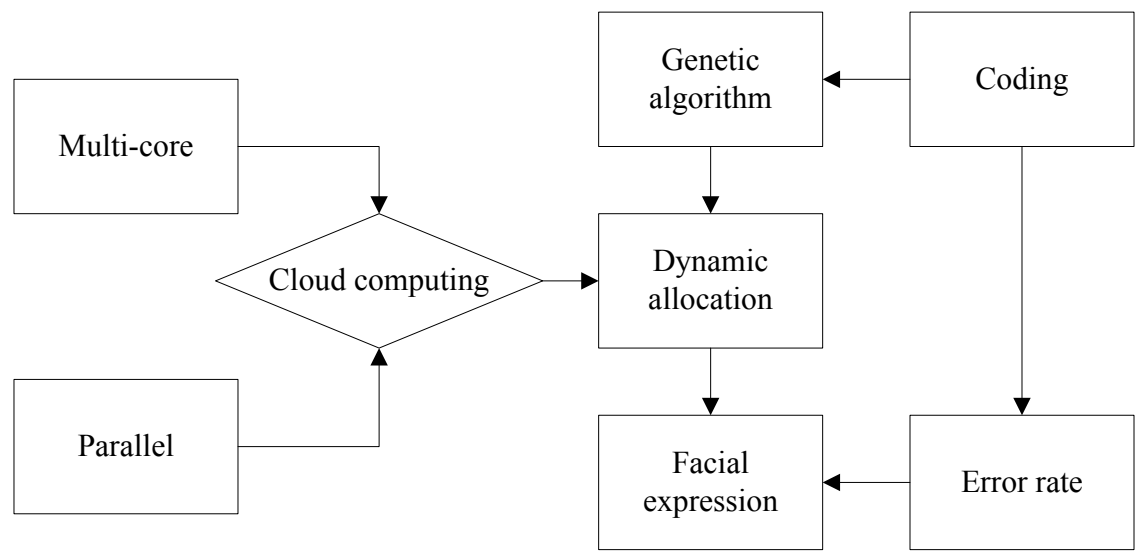

Fig. (1). The basic framework of facial expression recognition system.

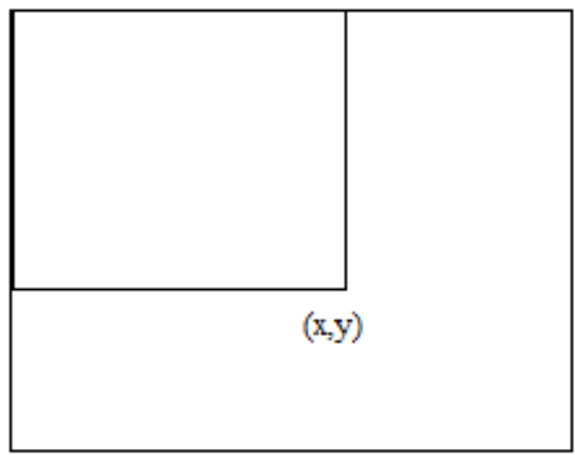

Fig. (2). Schematic diagram of integral image

finally it is indicated that the best expression recognition effect is Gabor+SVM and Haar+Adaboost [9]. Therefore, a facial expression recognition method based on the framework of Haar+Adabosst face recognition has very strong practicability. In 2010 Liu Qingshan et al. proposed a facial expression recognition method based on component characteristics of facial motion unit. Through the verification of expression database, this algorithm has more advantages than the general Haar+Adaboos method [10]. But there are some defects on selecting component characteristics of facial motion unit, so facial motion unit combination characteristics are proposed instead of component characteristics.

\subsection{The Extracting Method of Facial Motion Unit Com- bination Characteristics}

In the classic face recognition, Viola uses three types fo Haar features, also known as rectangle feature. The number of rectangle features is very large, if computing directly it will expend very large computational resources, therefore it needs to introduce the concept of integral graph, as shown in Fig. (2).

As shown in Fig. (2), it uses pixels point of the left rectangle of and $(x, y)$ to define integral image,

$t t(x, y)=\sum_{x^{\prime}<z, y^{\prime}<y} t\left(x^{\prime}, y^{\prime}\right)$ $s(x, y)=s(x, y-1)+t(x, y)$

Where $s(x, y)$ is the rows accumulating of pixels. For the convenience of calculation, it can initialize iteration, $s(x,-1)=0$ and $t t(-1, y)=0$. The method can be used to quickly compute the integral image, which can extract the facial unit fast. Based on the facial motion unit composition characteristics expression recognition method, it improves classical Haar+Adaboost algorithm, and it is realized through two parts, the first part is the main character integration, each integration characteristic is relative to a set of facial motion unit. The main steps of the algorithm are as follows [11]:

(1) Collecting the sample image and extract local Haar feature. $p$ isl e local area number, $p=1,2, \cdots, 49$;

(2) using the expression classifier supporting vector machine, so error rate of each local feature is $c$;

(3) Selecting the $n$ features with minimum error rate to compose new combination feature $Z$;

(4) The rest of local features uses face classifier of supporting vector machines, if the error rate is reduced, then continue to join the combination characteristic $Z$;

(5) Repeat the step(4), when the error rate is the lowest, record the combination characteristic $Z$. 


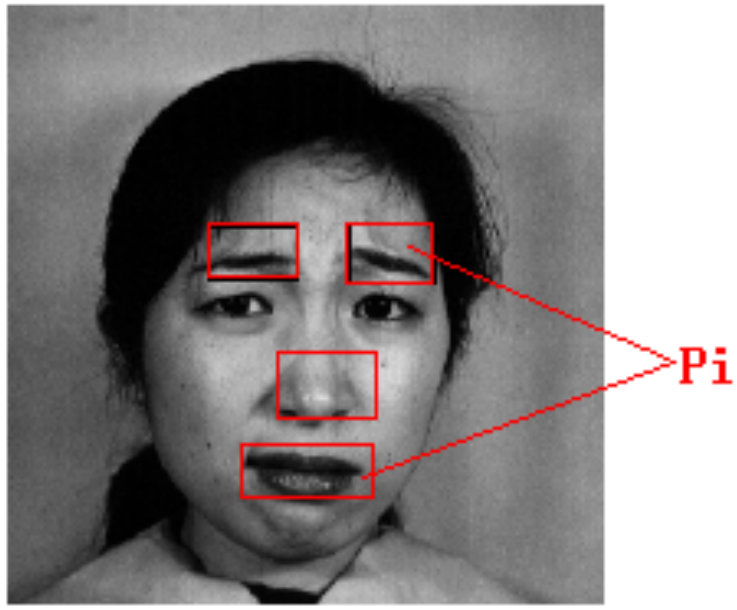

Fig. (3). Sample of combined feature set.

Fig. (3) shows the schematic diagram of the combined features. The second part is to use Adaboost to train these feature groups, finding really suitable for feature group of facial expression classification [12]. The main procedure of the algorithm is as follows:

(1) Image capturing and calibration each sample;

(2) Initialize weight value $w_{t}(t)=1 / N$;

(3) For $t=0, \cdots, n$ do;

(4) Running the algorithm 1, identify a set of combined feature;

(5) Training weak classifier $h_{1}$, and calculate the error rate $c$;

(6) Selecting $c 1=1 / 2 \frac{1-c}{c}$, update $D_{i}(i)$ :

$D_{t+1}(t)=\frac{D_{i}(i) \exp \left(-y_{i} a_{i} h_{i}(i)\right)}{Z_{i}}$, in which $Z_{t}$ is a normalized variables;

(7) End for;

(8)Output the final classifier:

$H(x)=\sum_{t}^{T} a_{t}, h_{t}(x)$

After Adaboost training and selection, it can identify the composition characteristics of the facial motor unit.

\subsection{Improved Facial Motion Unit Combination Feature Extracting}

The improved algorithm uses genetic algorithm to code, and mark the facial organs area effectively, which improves the training speed of sample classification. The feature extraction methods and steps of the improved are as follows [13]:
(1) Image capturing and calibration each sample;

(2) Initialize weight value $w_{t}(t)=1 / N$;

(3) For $t=0, \cdots, n$ do;

(4) Using genetic algorithm to find a set of combined feature, $v$ is facial expressions combined feature, $t$ is cross times, $a$ is the initial feature, based on the immune genetic algorithm it first generates a genetic sequence:

$$
\left\{v_{t-1}, v_{t-1}^{1}, v_{t-1}^{2}, \cdots, v_{t-1}^{n}\right\}, a_{t-1} \in R
$$

The initial vector $\left\{a_{l}^{1}, a_{l}^{2}, \cdots, a_{l}^{n}\right\}$ is as the initial chromosome. If each combination feature sequence $\left\{k_{j}^{1}, k_{j}^{2}, \cdots, k_{j}^{n}\right\}(j=1,2, \cdots, L)$ is a function of the expected output effect $\psi_{i}(i=1,2, \cdots, z)$, each composite feature sequence is sorted as follows:

$$
\begin{aligned}
& \operatorname{eval}\left(\psi_{m}\right)=\beta(1-\beta)^{i-1}, \\
& (i=1,2, \cdots, z), 0<\beta<1
\end{aligned}
$$

(5) Using immune genetic algorithm to do adaptive training weak classifier of proportional coefficient $h_{1}$, and calculating error rate $c$, as shown in formula (6)

$b_{c}=\sum_{m=1}^{c} \operatorname{eval}\left(\psi_{m}\right)(c=1,2, \cdots, z)$

Copying $Z$ chromosome, and do crossover operation on every initial combination characteristics $\left\{\alpha_{e}^{1}, \alpha_{e}^{2}, \cdots, \alpha_{e}^{n}\right\}$ and $\left\{\alpha_{f}^{1}, \alpha_{f}^{2}, \cdots, \alpha_{f}^{n}\right\}$.

$\bar{\alpha}_{e}^{j}=y \alpha_{e}^{j}+(1-y) \alpha_{e}^{j}$
$\bar{\alpha}_{f}^{j}=(1-y) \alpha_{f}^{j}+y \alpha_{f}^{j}, j=1,2, \cdots, n$ 
Where $x$ is a random real numbers, $x \in[0,1]$. Do mutate operation on the combination features, such as shown in formula (8).

$$
\left\{\bar{\alpha}_{e}^{1}, \bar{\alpha}_{e}^{2}, \cdots, \bar{\alpha}_{e}^{n}\right\}=\left\{\alpha_{e}^{1}, \alpha_{e}^{2}, \cdots, \alpha_{e}^{n}\right\}+N \cdot s
$$

(6) Doing crossover mutation operation through genetic algorithm $c 1=1 / 2 \frac{1-c}{c}$, and update $D_{i}(i)$ :

$$
D_{t+1}(t)=\frac{D_{i}(i) \exp \left(-y_{i} a_{i} h_{i}(i)\right)}{Z_{i}} \text {, in which } Z_{t} \text { is a normal- }
$$
ized variables;

(7) End for.

\subsection{Expression Feature Extraction}

Extracting the characteristics of local facial motion unit after the genetic algorithm encoding, the specific steps are as follows [14]:

(1) First assume the pixels of the image is $p \times p$, the horizontal line of the eye as a benchmark, moving step of window set to $p / 8$ pixel. The initial position of the sub window scanning is $p / 8$ in vertical direction, extracting five $\frac{p}{4} \times \frac{p}{4}$ sub windows, constructing sub block set of ocular movement unit, denoted by $\left\{P_{i}\right\}, i=1,2,3,4,5$, and through the genetic algorithm code it is marked.

(2) Constructing the export department of regional motion unit using the same method of sub blocks set, denoted as $\left\{Q_{i}\right\}, i=1,2,3,4,5$, and through the genetic algorithm code it is marked.

(3) In order to focus on the horizontal line of mouth and eyes as a benchmark, in the same way it extracts 3 sub windows as sub block set of nose movement area, denoted as $\left\{W_{i}\right\}, i=1,2,3$, and through the genetic algorithm code it is marked, total of 13 region extraction.

After the extraction of moving unit, combined with the crossover and mutation feature of genetic algorithm, calculating the Haar features of each sub block region using feature template.

\section{THE SUPPORTING VECTOR MACHINE EXPRES- SION CLASSIFIER}

\subsection{The Principle of Support Vector Machine}

For nonlinear problems, through the nonlinear transform, the training data is transformed into a linear problem in high dimensional space to obtain the optimal classification face [15]. Suppose there is a nonlinear mapping $\Phi: R^{d} \rightarrow H$. Training algorithm uses only a point in a space product, according to the functional theory, if the function satisfies the equation (1), it corresponds to a space inner product.

$\iint K\left(x_{i}, x_{j}\right) \psi\left(x_{i}\right)(x) d x d y>0, \psi(x) \neq 0, \int \psi^{2}(x)<\infty$

While the computational complexity is not increased, then the target function becomes :

$Q(a)=\sum_{i=1}^{n} a_{t}-\frac{1}{2} \sum_{i, j=1}^{n} a_{t}, a_{j} y_{i} y_{j} K\left(x_{i} x_{j}\right)$

And the corresponding classification function becomes :

$f(x)=\operatorname{sgn}\left[\sum_{i=1}^{n} a_{i} * y_{i} K\left(x_{i}, x\right)+b^{*}\right]$

Formula (11) is the support vector machine. The training sample data is mapped to high dimensional space, so as to achieve the process of linear separating.

\subsection{Improved Support Vector Machine Expression Clas- sifier}

The essential of support vector machine expression classifier is a two class classifier, but in practice it often needs to solve multi class classification problems, such as the given training set:

$X=\left[\left(x_{1}, y_{1}\right), \ldots,\left(x_{l}, y_{l}\right)\right] \in(x \times y)^{l}$

Where $x \in R^{d}, y=\{1,2, \ldots, N\}, i=\{1,2, \ldots, n\}$. For a decision function $f(x): x=R^{d} \rightarrow y$, carry out the following operations: $j$ class as the positive class, the rest of $N-1$ class as the negative class, decision function can be obtained by support vector machine.

$$
\begin{aligned}
& f^{j}(x)=\operatorname{sgn}\left(g^{j}(x)\right) \\
& g^{j}(x)=\sum_{i=1}^{l} y_{i} a_{i}^{j} K\left(x, x_{i}\right)+b^{j}
\end{aligned}
$$

Finally according to the multi classification decision function, it can judge that what kind of the test sample belongs to:

$$
f(x)=\arg \max _{1 \leq i \leq n} g^{j}(x)
$$

In order to improve the classification calculation speed, it can improve by the algorithm using cloud calculating collaborative simulation principle. Firstly training set in the solution domain is equidistantly segment. In the time domain $\left[t_{i}, t_{i+1}\right]$, it can build time generalized matrix $a_{1}, a_{2}, a_{3}, a_{4}$ and training set generalized matrix $b_{1}, b_{2}, b_{3}, b_{4}$. Generalized matrix for each coordination unit can be defined:

$\left\{\begin{array}{c}\mathrm{M}(m, n)=a_{1}+a_{2} m+a_{3} n+a_{4} m n \\ N(m, n)=b_{1}+b_{2} m+b_{3} n+b_{4} m n\end{array}\right.$ 


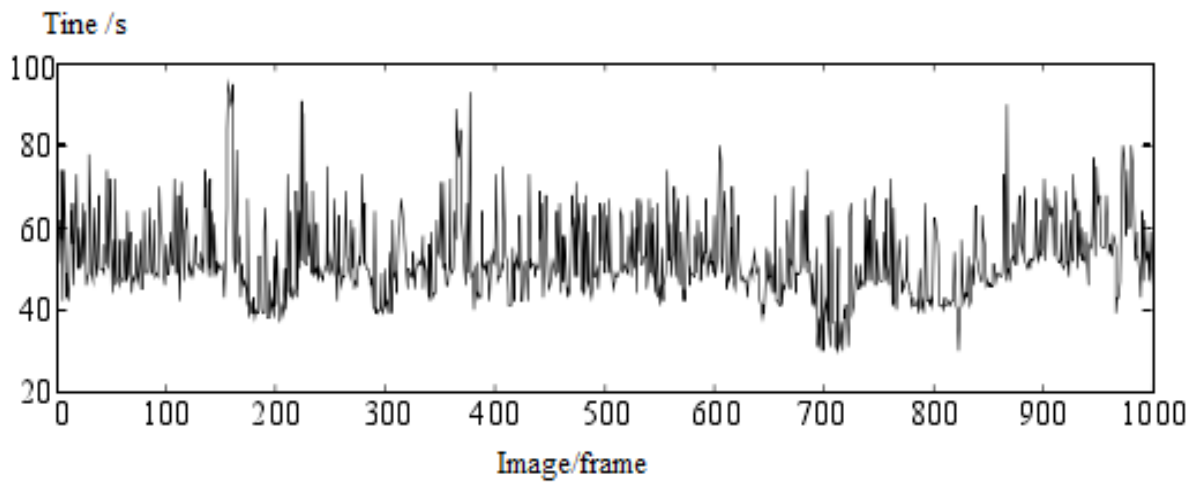

Fig. (4). Real-time test result of face accurate location algorithm.

Assumptions the classification results can be defined:

$X^{T}=\left[\begin{array}{llllllll}x_{1} & x_{2} & x_{3} & x_{4} & y_{1} & y_{2} & y_{3} & y_{4}\end{array}\right]$

Formula (16) and (17) can be written in matrix form:

$x(m, n)=\alpha a$

Formula (18) is established for classifying each collaborative simulation, so it can be obtained:

$\bar{X}=X a$

$H=\left[\begin{array}{cc}\bar{H} & 0 \\ 0 & \bar{H}\end{array}\right], \bar{H}=\left[\begin{array}{cccc}1 & a_{1} & b_{1} & a_{1} b_{1} \\ 1 & a_{2} & b_{2} & a_{2} b_{2} \\ 1 & a_{3} & b_{3} & a_{3} b_{3} \\ 1 & a_{4} & b_{4} & a_{4} b_{4}\end{array}\right]$

So the generalized coordinate $a$ is:

$a=H^{-1} \bar{x}$

$H^{-1}=\left[\begin{array}{cc}\bar{H}^{-1} & 0 \\ 0 & \bar{H}^{-1}\end{array}\right]$

Classification matrix of computer collaborative computing can be expressed as:

$S(m, n)=\alpha H^{-1}$

Through cloud computing collaborative simulation it can effectively improve the training speed of the separator, and make the classifier more powerful.

\section{EXPERIMENTAL RESULTS AND ANALYSIS}

This paper uses the classical Jaffe face database to carry on the simulation experiment. The database contains 7 types of expression; every kind of expression has some pictures, a total of 213 face images, containing 10 different women images. First, keep one of each expression, and the rest of the pictures are done genetic algorithm coded and as the training sample, then the rest of each expression pictures as test samples, finally using support vector machine classifier to recognize and classify facial expression.

In order to calculate the accuracy rate of recognition, this paper adopts MATLAB programming to carry on the statistics on the recognition results. But MATLAB is strong professional software, in order to improve the easy operation; it uses VB calling method to do visualization. There are many kinds of ways in the VC environment calling MATLAB, now the MATLAB program $m$ files to dynamic link library for the $\mathrm{C}++$ calling, and MATLAB command is: $m c c-t-W$ lib:libfilename -T link:lib filename. libfilename is a dynamic link library file name from compiler; filename is name of $m$ file, and the $m$ file must be a function. After command, libfilename.lib, libfilename.dll and libfilename.h files are obtained, and the main procedures are as follows:

$$
\begin{aligned}
& \text { x_ba=mean(xi); } \\
& \text { y_ba=mean(Y); } \\
& \text { F_alpha=input('>>>>>>Input minimum error } \\
& \text { while } \sim(\text { isscalar(F_alpha) \&\& F_alpha }<1 \text { \&\& } \\
& \text { F_alpha=input('Input training times, } \alpha=\text { '); } \\
& \text { end } \\
& \text { F_fenweidian=finv(1-F_alpha,k,n-k-1); } \\
& \text { c=k/(n-k-1)*F_fenweidian; } \\
& \text { if c_flag }>\text { c }
\end{aligned}
$$$$
\text { F_alpha }=\operatorname{input}('>>>>>\text { Input minimum error rate } \mathrm{a}=\text { '); }
$$$$
\text { while } \sim(\text { isscalar(F_alpha) \&\& F_alpha }<1 \& \& \text { F_alpha }>0)
$$

This paper selects 800 face images for face recognition test from the image sequence. Firstly it verifies the face location and local characteristics combination coding, and does real-time test experiment on the positioning algorithm, the experimental results are as shown in Fig. (4).

It can be seen from Fig. (4), the single frame image positioning average consumes only $52.3 \mathrm{~ms}$, and it is obvious that this algorithm has better real-time. Through tests on seven kinds of expressions, the results are obtained in Table $\mathbf{1 .}$ 
Table 1. Test results of the same individual facial expression recognition success ratio.

\begin{tabular}{|c|c|c|c|c|c|}
\hline & Test 1 /\% & Test 2 /\% & Test 3 /\% & Test 4 /\% & Test 5 /\% \\
\hline \hline Anger & 91.26 & 90.02 & 95.38 & 89.19 & 75.31 \\
\hline Happy & 96.82 & 96.81 & 90.62 & 79.07 & 93.22 \\
\hline Neutral & 82.82 & 81.41 & 80.73 & 93.20 & 89.83 \\
\hline Surprised & 93.78 & 89.41 & 91.18 & 81.52 & 94.21 \\
\hline Hate & 91.33 & 93.12 & 87.02 & 89.11 & 93.25 \\
\hline Fear & 89.78 & 85.74 & 89.33 & 94.10 \\
\hline Sadness & 80.12 & 94.92 & & 93 \\
\hline
\end{tabular}

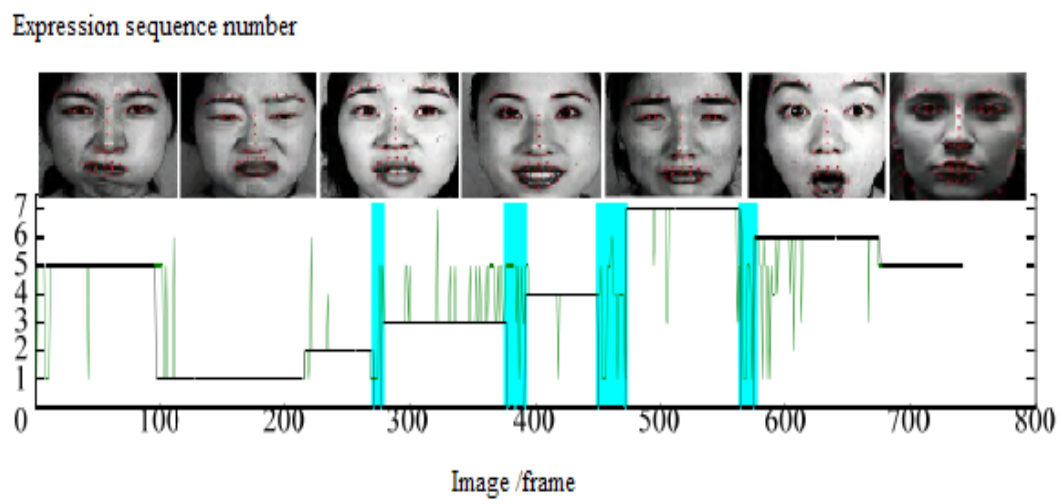

Fig. (5). The average statistical results for the same individual expression recognition.

Table 2. Test results for different individual facial expression recognition success ratio.

\begin{tabular}{|c|c|c|c|c|c|}
\hline & Test 1 /\% & Test 2 /\% & Test 3 /\% & Test 4 /\% & 94.23 \\
\hline \hline Anger & 91.12 & 90.32 & 93.21 & 97.23 & 95.22 \\
\hline Happy & 97.32 & 98.13 & 95.26 & 90.23 & 99.25 \\
\hline Neutral & 93.13 & 92.28 & 91.32 & 94.11 & 80.58 \\
\hline Surprised & 94.28 & 92.36 & 81.19 & 83.13 & 85.11 \\
\hline Hate & 82.21 & 83.23 & 87.12 & 83.71 \\
\hline Fear & 86.25 & 85.78 & 83.12 & 82.12 \\
\hline
\end{tabular}

It can be seen from Table 1, for the same individual expression, 7 basic facial expression recognition rates is higher than $80 \%$, and the system has higher recognition rate on angry, happy, neutral, surprise expression. This is related to the local facial feature, when there is such an expression, the relative position and shape changes obviously for human face sensitive. And hate, fear and grief expressions have lower recognition rate, as the relative position and shape change of face sensitive area is not obvious.
Fig. (5) is the average success recognition rate for the same individual basic facial expression. It can be seen from the graph, the system has higher average recognition rate for angry, happy, neutral, surprised than the disgust, fear and sadness, and the results are the same as the test results of monomer.

From Table 2 it can be seen, for different individual expression recognition test, 7 kinds of basic expression 
Expression sequence number

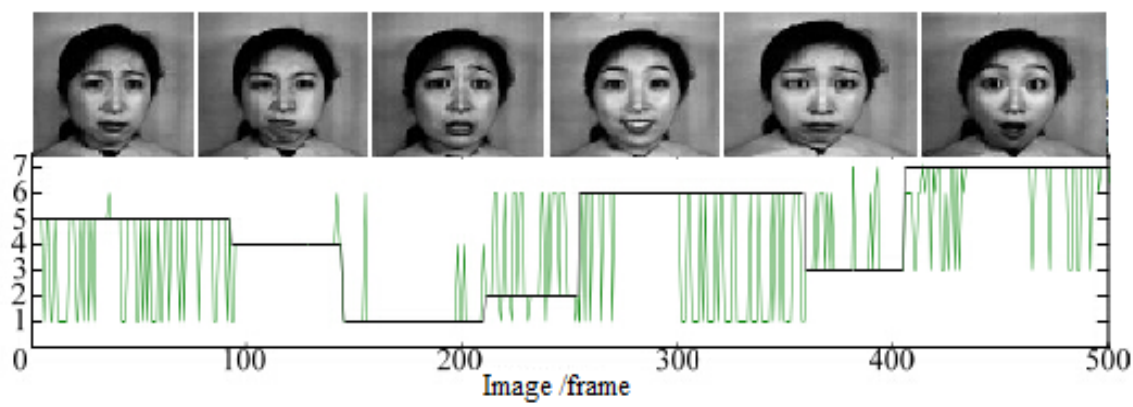

Fig. (6). The average statistical results for the different individual expression recognition.

recognition rate is not less than $80 \%$, and it is the same as the individual test results trend. The system has higher recognition rate on angry, happy, neutral, surprise, and lower recognition rate on disgust, fear and sadness.

Fig. (6) is the average recognition success rate for different individual basic expressions. From the figure it can be seen, the system has higher recognition rate for angry, happy, neutral, surprised than the four kinds of expression of disgust, fear and sadness, and the trend is the same as statistics of different individuals, which verified the reliability of the algorithm.

\section{CONCLUSION}

This paper uses genetic algorithm to improve extraction method of facial motion unit combining feature, and does genetic algorithm coding on facial organ region, avoiding the error using map feature training, which improves the accuracy of feature extraction. This paper uses cloud calculation collaborative simulation principle to improve the classifier and the training set is done equidistantly segmentation in the solution domain, establishing the generalized matrix of facial local feature, enhancing classification function. Through the testing of the expression database, the improved method has higher recognition ratio on facial expression of different individuals and the same individual, faster calculation speed and better performance.

\section{CONFLICT OF INTEREST}

The authors confirm that this article content has no conflicts of interest.

\section{ACKNOWLEDGEMENTS}

Declared none.

\section{REFERENCES}

[1] Z. L. Bai, and X.X. Li, "The report editor application system based on Struts framework," Computer System Application, vol.2, no.3, pp. 56-59, 2014.

[2] H.T. Yan, L. Wang, K.M. Li, and J.F. Liu, "Face recognition fusion MBP and EPMOD," Journal of China Image Graphics, vol.2, no.1, pp. 42-45, 2014.

[3] X. H. Ma, and Y. Q. Tan. "Face recognition algorithm based on the embedded parity preserving," Acta Automatica Sinica, vol.3, no.1, pp. 12-15, 2014.

[4] Y.F. Li, and L.C. Li, "Application, study status and key problems of affective computing in network distance education system," Modern Distance Education, vol.2, no.4, pp. 68-70, 2012.

[5] Q. Y. Wu, Y.Q. Zhang, Q.H. Zheng, and Y. Fu, "Quantitative evaluation for E-Learning user psychological experience," Journal of Xi'an Jiao Tong University, vol.2, no.12, pp. 89-91, 2012.

[6] Z. K. Han, "Design and realization of examinee identity authentication system based on face recognition technology," Computer and Digital Engineering, vol.5, no.1, pp. 112-115, 2012.

[7] J. Zhang, Y.Z. Zhan, Q.R. Mao, and X. Zou, "The facial expression recognition based on Gabor wavelet and sparse representation," Computer Engineering, vol.5, no.6, pp. 142-145, 2012.

[8] F. L. Wang, and H. B. "Facial expression feature extraction based on Gabor wavelet,' Electronic Design Engineering, vol.5, no.3, pp. 22-25, 2012.

[9] N. Liu, H. Su, C. H. Guo, and J. Zhou, "The improvement of eye location method based on the Hough transform," Computer Engineering and Design, vol.5, no.4, pp. 66-70, 2011.

[10] B. Jiang, K.B Jia, and G.S. Yang, "Research progress of facial expression recognition," Computer Science, vol. 5, no.4, pp. 87-89, 2012.

[11] M. Zhong, and H. F. Xue, "Facial expression recognition fusion Garbor wavelet and manifold learning," Measurement and Control Technology, vol. 2, no. 12, pp. 42-45, 2013.

[12] S. Li. "The impact of cognitive on emotional research," Science Education, vol. 5, no. 2, pp. 56-58, 2011.

[13] H. Y. Wang, X. Li, "Improved Gabor and ADABOOST facial expression recognition," Journal of Liaoning University of Technology (Natural Science Edition), vol.5, no.1, pp. 45-48, 2012.

[14] N. Ni, and G. Lu, "Facial expression recognition based on video," Computer Engineering and Application, vol.4, no.17, pp. 55-58, 2013.

[15] Y. H. Lv, and J. X. Cui, "Facial expression recognition based on FWT-Projection-BPNN," Journal of Northeast Normal University (Natural Science Edition), vol.5, no.2, pp. 85-89, 2012. 